\title{
Fractal Design of Multiband and Low Side-Lobe Arrays
}

\author{
Carles Puente-Baliarda, Member, IEEE, and Rafael Pous, Member, IEEE
}

\begin{abstract}
Most array factor design techniques are highly dependent on the operating wavelength. In this paper, a novel technique based on fractal structures is described for multiband operation. The analysis is focused in two different approaches: the fractal spatial arrangement of array elements and the fractal design of array factors. Although the patterns of fractal arrays show some interesting similarity properties at several bands, the directivity is not held constant through the bands. Nevertheless, such structures have been shown to be useful for designing low side-lobe arrays with equally weighted current elements. On the other hand, the fractal array factors presented do keep the same shape at several bands because they are designed as selfsimilar curves. The arrays that would synthesize such patterns present a characteristic power-law current distribution analogous to the spectral distribution of the bandlimited fractal Weierstrass function.
\end{abstract}

\section{INTRODUCTION}

$\mathbf{T}$ HE main problem with the design of wideband or frequency-independent radiating systems is that once the system is designed to match its characteristic size to the operating wavelength, its parameters will change when operating at different wavelengths. For single antennas, this characteristic size is the length of the antenna. For arrays, the spacing between elements is also important.

This constraint has been present in the development of all current frequency-independent antennas. Angles, cones, and spirals are examples of some shapes that have successfully been used to design frequency-independent antennas [1]-[4]. All of them have a common characteristic: their shape is invariant under a scaling transformation or, in other words, their shape relative to all wavelengths is constant. Similarly, log-periodic dipole arrays [5] are designed with dipoles of several lengths and a variable spacing between elements in such a way that the structure keeps the same shape under some scaling transformations as well.

Fractals are self-similar structures. This means that their shape remains the same under a change of scale. They are said to possess no characteristic size [6]-[8]. Hence, if they could be used in the design of radiating systems, one would expect of them a multiwavelength operation. This paper analyzes two possible approaches to the fractal design of multifrequency arrays. First, fractal spacing between array elements is analyzed (Section II). Second, a fractal design of array patterns is

Manuscript received May 16, 1994; revised August 21, 1995. This work was supported by the Spanish Commission of Science and Technology under Grant MAT95-1038-C02-02.

The authors are with the Department of Signal Theory and Communications, Polytechnic University of Catalonia, Barcelona, Spain 08071.

Publisher Item Identifier S 0018-926X(96)03232-2. introduced and the relative current distribution of such arrays is derived (Section III). Although the fractal arrays analyzed in Section II present some interesting similarity properties (the array factor has a similar shape at several frequencies), the directivity and the main-lobe width is not held constant with frequency due to fractal truncation. Nevertheless, such a fractal distribution of the array elements have shown some other interesting properties: they can be used to synthesize low side-lobe radiation patterns with a uniform current distribution of elements within the array. That is, some approximations to common low side-lobe radiation patterns, like those corresponding to triangular and binomial distributions, can be achieved by placing a set of equal amplitude array elements according to the same algorithm used to generate fractally spaced arrays.

The undesired changes in the pattern parameters of the analyzed fractal arrays has led the research to the design of the fractal patterns in Section III. In this section, the design is focused on the definition of the array factor. A well-known set of fractal curves, the Koch curves, are used to describe patterns that keep the same shape at different scales. When the visible range of the array factor is modified by means of a change in the array operating frequency, the array radiates through a scaled version of the whole array factor with the same directivity and lobe profile. The arrays that generate such fractal patterns have been shown to have power-law current distributions which present some interesting scaling properties as well [9]-[11].

\section{FRACTAL ARRAYS}

Array factors are highly dependent on the operating wavelength. An increase in the operating frequency is translated into an expansion of the visible range. This means that grating lobes will appear in the radiation pattern at high enough frequencies. This is a strong inconvenience because although many different techniques based on an ideal current feeding of each array element allow us to synthesize array factors with a desired directivity or side-lobe ratio (SLR), the design is frequency dependent. Most of these techniques assume a uniform spacing between elements which becomes the main bandwidth limiting factor.

In this section, a nonuniform fractal spacing for the element distribution of an antenna array will be analyzed. An ideal current feeding system to each array element is assumed, as usual, in array theory. Kim and Jaggard [10] first proposed a nonuniform random fractal spacing for improving the SLR of random arrays in 1986. Also, in 1992, Jaggard et al. 
showed how the diffraction pattern from a triadic Cantor target presented some interesting self-similarity properties with increasing growth stages. Here, a deterministic fractal array designed by placing the array elements at the points of a generalized Cantor set is introduced.

Although other fractal structures could be used for designing arrays, this particular one has been chosen for the analysis because it provides a simple, well-known fractal set of discrete points that can describe a linear array. A bandlimited [9] version of the fractal structure can be constructed iterating several convolutions which leads to an analytical expression for the array factor, providing a compact way of deriving the properties of the resulting array. One of the characteristic features of the Cantor set and other fractal structures is that they contain an infinite number of subsets at different scales which are all identical to the whole set. Thus, if an array is built by placing the elements at the points of the Cantor set, one could expect these smaller substructures to radiate at shorter wavelengths in the same way that the whole structure radiates at longer wavelengths.

To analyze the behavior of an array based on such a fractal, let us first point out an alternative procedure to generate the Cantor set. The procedure starts by taking two delta functions, spaced a distance $d$ in the $z$ axis, as the basic structure (usually known as generator in fractal terminology [8], [9]). Then, the generator is scaled by a factor of three to obtain another structure composed by two delta functions spaced $d / 3$. If one convolves these two structures, a set of four delta functions will be placed at the points of a Cantor set constructed with only two iterations. It can be seen that this convolution could be iterated an infinite number of times to obtain the complete set. That is, if we call $f(z)$ the two delta function generator, the whole Cantor set $c(z)$ can be written as

$$
c(z)=\cdots f(z) * f(3 \cdot z) * f(9 \cdot z) \cdots * f\left(3^{n} \cdot z\right) \cdots .
$$

It should also be noted that this procedure can be carried out with a different generator (with an arbitrary number of delta functions) and with a different scaling factor $\delta$. In this case, a generalized Cantor structure (hereafter, Cantor array) can be defined as follows:

$$
c(z)=\stackrel{\infty}{\mathrm{X}}_{n=-\infty}\left\{f\left(z \cdot \delta^{n}\right)\right\}
$$

where $X$ symbolizes the convolution operator. The structure just defined is a further generalization of the Cantor bar introduced by Sun and Jaggard in [16] and covers from the original Cantor set to the triangular Cantor array and more sophisticated structures.

The array factor corresponding to $c(z)$ can be written in terms of the Fourier transform of the generator $F(\psi)$ as

$$
\begin{aligned}
C(\psi)= & \cdots \delta^{2} \cdot F(\delta \psi) \cdot \delta \cdot F(\delta \psi) \cdot F(\psi) \\
& \cdot \frac{F\left(\frac{\psi}{\delta}\right)}{\delta} \cdot \frac{F\left(\frac{\psi}{\delta^{2}}\right)}{\delta^{2}} \ldots \\
= & \prod_{n=-\infty}^{\infty} F\left(\frac{\psi}{\delta^{n}}\right)
\end{aligned}
$$

where $\psi$ is defined as usual in array theory as

$$
\psi=k d \cos \theta+\beta
$$

with $d$ being the spacing of the generator array, $\theta$ the angle between the direction of propagation and the axis of the array, $\beta$ the progressive phase-shift of the generator array, and $k=2 \pi / \lambda$ the wave number. This alternative way of generating the fractal and deriving its array factor can give a physical insight on the modulation effects on the patterns first suggested in [11]. That is, the resulting array factor (3) can be obtained by repeatedly modulating (multiplying) the array factor of the generator with a scaled version of itself.

It can be seen that a frequency change by a factor of $r$ implies a proportional scaling of both the $\psi$ parameter and the array factor $C(\psi)$. That is, $C(\psi)$ becomes

$$
\begin{aligned}
C(r \psi)= & \cdots F(r \delta \psi) \cdot F(r \psi) \\
& \cdot F\left(\frac{r \psi}{\delta}\right) \cdot F\left(\frac{r \psi}{\delta^{2}}\right) \cdots F\left(\frac{r \psi}{\delta^{n}}\right) \\
= & \prod_{n=-\infty}^{\infty} F\left(\frac{r \psi}{\delta^{n}}\right) .
\end{aligned}
$$

Now, if the frequency shift $r$ is taken to be $\delta^{p}$ then the array factor will be

$$
\begin{aligned}
C\left(\delta^{p} \psi\right) & =\prod_{n=-\infty}^{\infty} F\left(\frac{\delta^{p} \psi}{\delta^{n}}\right) \\
& =\prod_{n=-\infty}^{\infty} F\left(\frac{\psi}{\delta^{n-p}}\right) \\
& =\prod_{m=-\infty}^{\infty} F\left(\frac{\psi}{\delta^{m}}\right) \\
& =C(\psi)
\end{aligned}
$$

which implies that the array factor generated by the Cantor structure is a $\log$-periodic (LP) function with a $\log$-period $\delta$. That is, in logarithmic scale

$$
C_{\log }[\log (\psi)]=C_{\log }[\log (\psi)+p \cdot \log (\delta)] \quad p \in \mathbb{Z} .
$$

The main conclusion derived from (7) is that the infinite Cantor array would have the same array factor at an infinite number of bands (which is a remarkable property not shared by uniform spaced arrays, even when infinite). Before going any further in our conclusions two important facts should be pointed out. First, this would be a multiband system and not a frequency-independent system, since (7) only implies that the behavior will be the same at several bands spaced by a factor of $\delta$, but does not imply a frequency-independent behavior within each band; however, a multiband behavior would be a significant improvement for an array design in applications, such as frequency hopping schemes in radar and spread spectrum communication systems. Second, this property would apply only to the infinite array. The corresponding bandlimited realization of the fractal structure will hold the similarity properties through as many bands as iterations used in the 


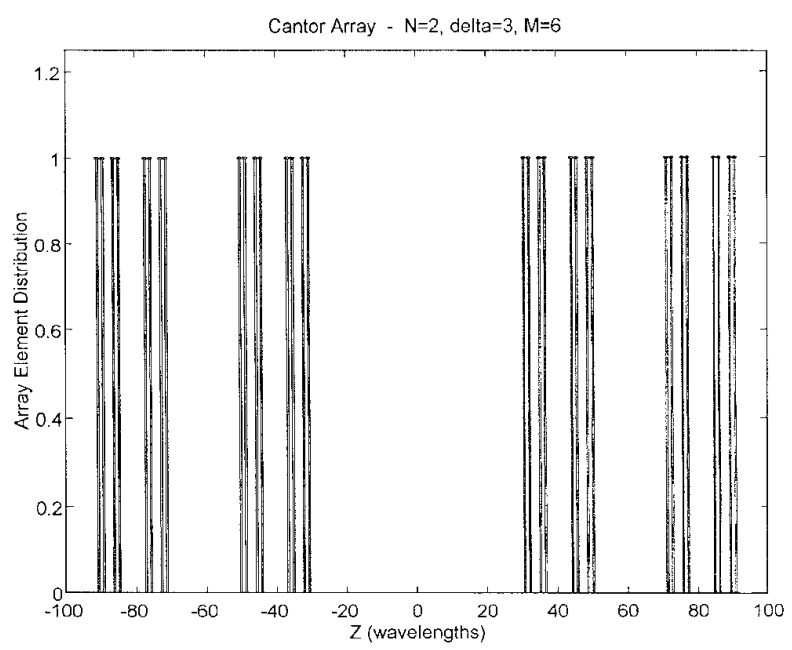

Fig. 1. Cantor array based on the classical Cantor set. The array has 64 elements and it is constructed from a two-element generator and a log period $\delta=3$.
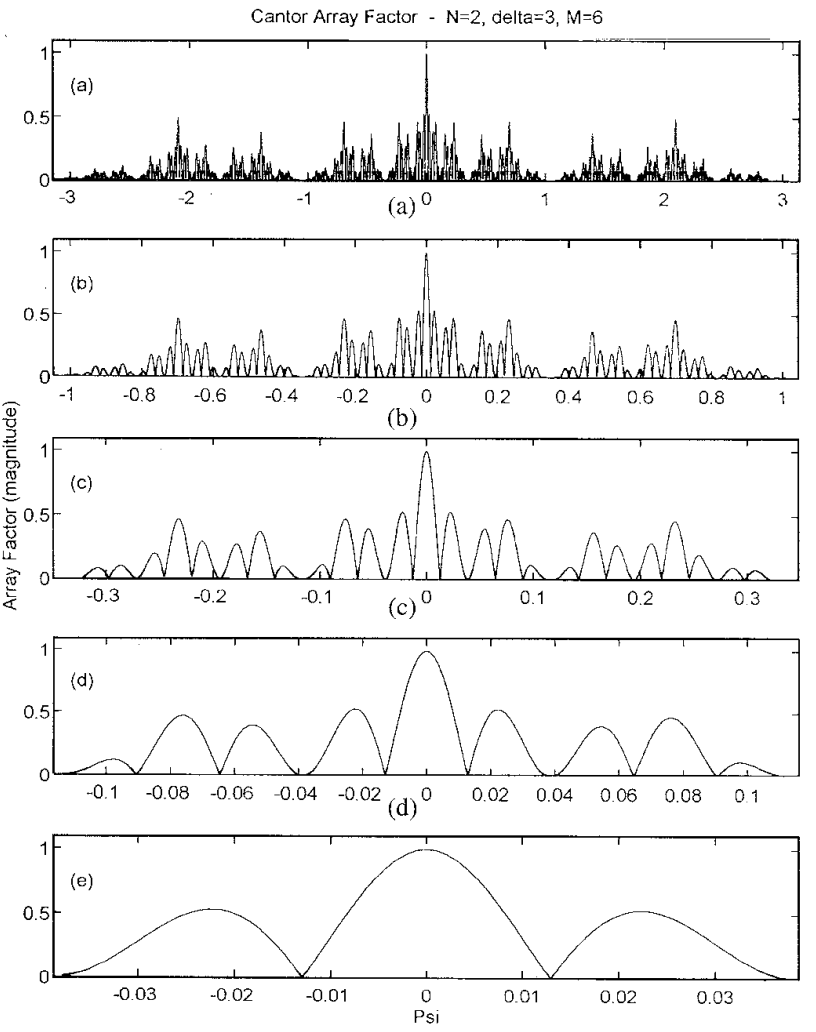

(e)

Fig. 2. Array factor for the Cantor array on Fig. 2. The array factor is plotted for five operating wavelengths: (a) $\lambda_{0}=\delta / 2$, (b) $\lambda_{1}=3 \lambda_{0}$, (c) $\lambda_{2}=9 \lambda_{0}$, (d) $\lambda_{3}=27 \lambda_{0}$, and (e) $\lambda_{4}=81 \lambda_{0}$. The similar lobe structure of the patterns at those frequencies can be noticed.

construction procedure, but not through an infinite set of bands. The example in Figs. 1 and 2 illustrates this fact.

The patterns on Fig. 2 present some interesting features. They look similar in the sense that they show a similar distribution of the main secondary lobes and that the SLR is kept almost constant at different wavelengths. This kind of similarity is analogous to the similarity at several fractal growth stages of the diffraction patterns shown in [11] for a fixed wavelength. One can explain this result by noticing that each time the wavelength is increased, the closest elements collapse into an equivalent single element from the radiation point of view. Hence, at each longer wavelength the equivalent array effectively loses one iteration or growth stage. If the fractal were ideal, it would keep exactly the same shape after collapsing, but since it is a bandlimited fractal, the array and its radiation patterns remain similar but not equal, at each frequency. Together with the array's high secondary lobes, this implies an inconvenient feature on the performance of the array: the main lobe width increases as the frequency is reduced.

The problem of the high secondary lobes of the pattern is related to an array characteristic known in fractal theory as lacunarity. A fractal structure is said to present high lacunarity when it has large gaps between the different fractal substructures [6], [7]. In terms of the array of Fig. 1, the gaps between subarrays are too large compared to the smallest operating wavelength which has been chosen to match twice the distance between two elements of the smallest substructure. To analyze the effect of the lacunarity and the $\log$ period in the pattern conformation, the analysis will be particularized to the simplest case-Cantor arrays constructed from a uniform element amplitude generator.

\section{A. One Particular Case of the Generalized Cantor Array: The Uniform Generator}

In the following, the analysis will be focused on the particular case of the generator function

$$
f(z)=\sum_{n=0}^{N-1} \delta\left(z-n d+\frac{N-1}{2} d\right)
$$

which represents a set of $N$-delta functions of equal amplitude, spaced a distance $d$ and centered at the origin. According to (3), the array factor for a Cantor array generated after $M$ iterations with the above generator can be written as

$$
C(\psi)=\prod_{n=0}^{M-1} \frac{\sin \left(N \frac{\psi}{2} \delta^{n}\right)}{N \sin \left(\frac{\psi}{2} \delta^{n}\right)}
$$

where $N$ is the number of elements of the generator in (8) and $\delta$ is the $\log$ period. As it will be shown in this section, the ratio $\delta / N$ is strongly related to the lacunarity of the fractal and defines some of the properties of the array factor. Also, this ratio can be related to the fractal dimension $D$ which can be calculated as [6], [9], [10], and [12]

$$
D=\frac{\log (N)}{\log (\delta)} \text {. }
$$

Equation (10) gives a fractal dimension of $D=0.63$ for the case presented in Fig. 2. At this point, the analysis will be focused in two cases, when $\delta$ becomes close to unity and when $\delta$ is larger than $N$. 


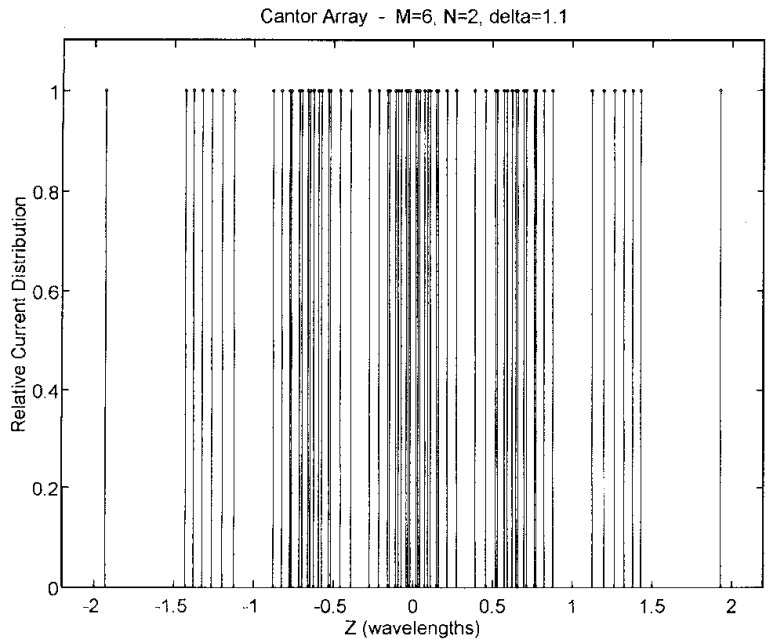

Fig. 3. Near-binomial array generated after six iterations from a two-element generator and a $\log$-period $\delta=1.1$. Although the array has a uniform-amplitude distribution of elements, its pattern is close to that of the binomial array.

The Binomial Array as a Particular Case of the Cantor Array: The particular case $\delta=1$ represents the convolution of $M$ equal distributions. In this case, (9) can be rewritten as

$$
C(\psi)=\left[\frac{\sin \left(N \frac{\psi}{2}\right)}{N \sin \left(\frac{\psi}{2}\right)}\right]^{M} .
$$

Thus, the binomial distribution can be obtained by taking a generator with only two elements, i.e., $N=2$ and a logperiod $\delta=1$. A larger number of elements in the generator will result in a triangular distribution for $M=2$ and a distribution that tends to a Gaussian shape for increasing values of $M$ (central limit theorem). In this later case $(N>2)$, the SLR in $\mathrm{dB}$ decreases linearly with the number of iterations, which is a convenient method for designing low-side lobe arrays. Nevertheless, this method has a great inconvenience-the dynamic range of the element amplitudes within the array is so large that small errors in the feeding network change the weight of smallest elements, distorting the final pattern.

Uniform-Amplitude, Nonuniformly-Spaced Arrays for NearBinomial Pattern Design: Now let us take a generator with two elements and chose a log period close to 1 , for instance $\delta=1.1$. One can expect the corresponding pattern to look very similar to the binomial one since the expression for the array factor (9) will be almost the same. Hence, although in this case the array loses its multiband properties, it presents a very interesting low side-lobe array factor. Also, there is a fundamental difference between the shape of this array and the binomial one. In the $\delta=1.1$ case, none of the elements of the $M$ convolving subarrays overlap, thus having a final array with a uniform-amplitude distribution over $2^{M}$ nonuniformlyspaced elements (Fig. 3).

The main feature of this array is that although the pattern is very close to the binomial one (SLR $<65 \mathrm{~dB}$ ), the amplitude distribution of the elements is uniform which greatly simplifies the feeding network. A uniform current amplitude distribution through all the array elements can be obtained by means of a combination of $\lambda / 4$ and $\lambda / 2$ transmission lines, regardless of mutual coupling effects [13]. Also, it can be seen that this scheme could be repeated to generate very close patterns to any of the family described in (11), but with uniform distributions of elements.

The graph in Fig. 4 can be used for the design of such nearbinomial, low side-lobe arrays. It represents the SLR on the array factor for several $\log$ periods and number of iterations $(M)$. It is interesting to remark that the side lobes are greatly reduced for log periods below 1.5. Also, the SLR tends to the same level regardless of $M$ when the $\log$ period is over $\delta=$ 2 , which is a logical result if one notices that above this value the patterns become self-similar at each growth stage [11]. When $\delta$ is below 2 the SLR is lower for a higher $M$ and the directivity of the array increases because the total length of the array is larger as well. Nevertheless, too many iterations will make the array structure denser and some elements will be placed very close to each other, which can make the physical implementation of the array complicated.

The Uniform Array as a Particular Case of the Cantor Array: The case under study is now $\delta=N$. Again, by taking the general expression in (9), the following expression can be derived:

$$
\begin{aligned}
C(\psi)= & \frac{\sin \left(N \frac{\psi}{2}\right)}{N \sin \left(\frac{\psi}{2}\right)} \cdot \frac{\sin \left(N^{2} \frac{\psi}{2}\right)}{N \sin \left(N \frac{\psi}{2}\right)} \\
& \ldots \frac{\sin \left(N^{M} \frac{\psi}{2}\right)}{N \sin \left(N^{M-1} \frac{\psi}{2}\right)} \\
= & \frac{\sin \left(N^{M} \frac{\psi}{2}\right)}{N^{M} \sin \left(\frac{\psi}{2}\right)}
\end{aligned}
$$

which is the array factor for a uniform distribution of $N^{M}$ elements. The construction of this uniform array as a convolution of $M$-uniform distributions at different scales can be seen again as a particular case of the process described in (2). The scaling parameter is such that at each iteration, the separation between subarrays is equal to the spacing between two elements of those subarrays. In contrast to the cases presented before, the SLR here does not change with the number of iterations, always keeping its value around $13 \mathrm{~dB}$.

Optimum Lacunarity for Self-Similar, Low Side-Lobe Fractal-Cantor Arrays: Let us now analyze the relationship between the lacunarity of the fractal structure and the SLR of the patterns for a more general case. As pointed out in (3), the Cantor array factors can be understood as a product of $M$ subpatterns at different scales. Each time the structure is convolved with the next wider scaled generator, the pattern is multiplied with a compressed version of the generator's array factor. By choosing a log period very close to $\delta=1$, as shown previously, the compression becomes very slight at 


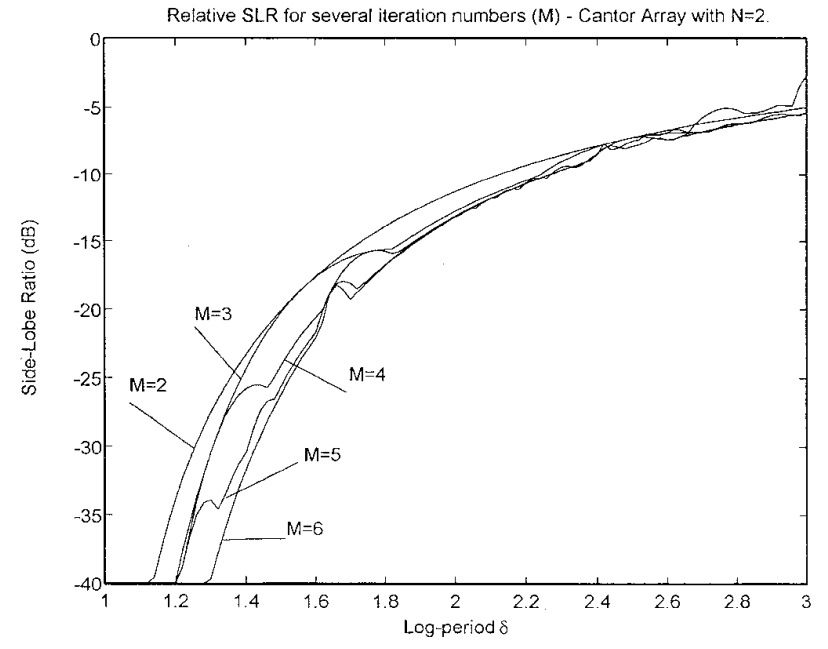

Fig. 4. Side-lobe ratio (SLR) as a function of the log-period $\delta$ of the two-element generator $(N=2)$ Cantor array. The graph is plotted for several iteration numbers $(M)$. Notice that for $\delta=2$ we have a uniform array with a SLR around $13 \mathrm{~dB}$, and that the SLR is greatly reduced for small $\log$ periods.

each iteration, resulting in a product of $M$-array factors very similar to the product expressed in (11). However, if $\delta$ is made large enough, the compression will be such that many grating lobes of the larger generator will appear within the visible range. The problem becomes specially important when $\delta>N$ and the array becomes self-similar at several bands. When $\delta=N$ the main grating lobe of a generator's array factor is placed on top of the first null of the previous generator's array factor. Equation (12) demonstrates that the product of these two sinc functions results in a narrower sinc function as well. Nevertheless, when the ratio $\delta / N$ is made larger than unity, the grating lobes appear within the main lobe of the previous array factor of the iteration. If these lobes fall near the maximum of this previous array factor, they will be greatly enhanced. In this case, the array presents a highly lacunar distribution and, consequently, large secondary lobes.

One can establish an upper bound to safely design fractalCantor arrays without increasing the side-lobe level above -13 $\mathrm{dB}$. By choosing a ratio $\delta / N=1.2$ (Fig. 5), the first grating lobe is placed at a $\psi$ point within the main lobe, such that when weighted by the amplitude of this main lobe, the grating lobe is reduced to the level of the first secondary lobe (i.e., 13 $\mathrm{dB}$ ). Therefore, one could state that Cantor arrays should be designed with a lacunarity such that

$$
\frac{\delta}{N}<1.2
$$

or equivalently with a fractal dimension

$$
\begin{aligned}
D & \geq \frac{\log (N)}{\log (N)+\log (1.2)} \\
& \approx \frac{\log (N)}{\log (N)+0.08} .
\end{aligned}
$$

It can be noticed that $D$ tends to unity for a large number of generator elements $N$. That makes sense when one takes into account that to avoid high secondary lobes the gaps between
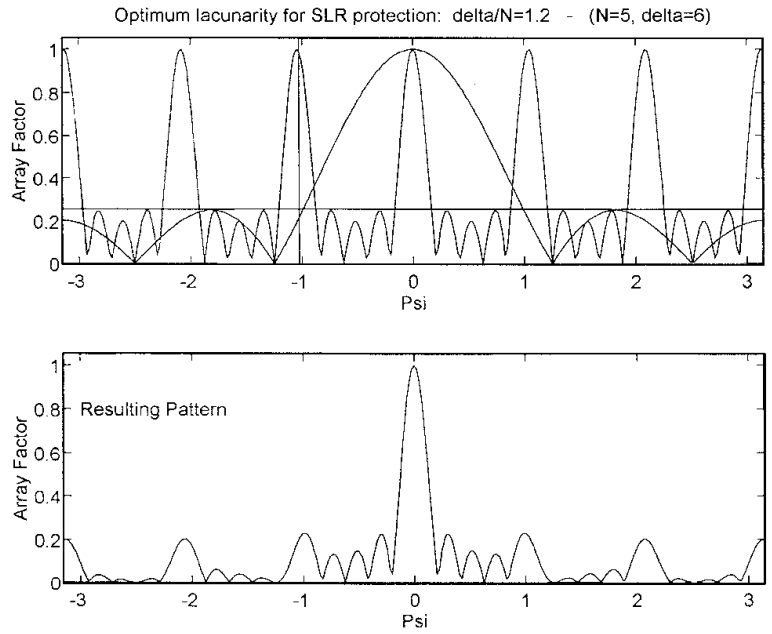

Fig. 5. The maximum ratio $\delta / N$ that does not increase the side-lobe level with respect to the uniform case is $\delta / N=1.2$. In such a case, the first grating lobe is located at a $\psi$ point where the main lobe has decayed $13 \mathrm{~dB}$, with respect to the maximum.

substructures must have a limited size relative to the shortest wavelength, regardless of the total number of elements within the array. Therefore, for a larger $N$ the elements of the array tend to fill a straight line more densely (one dimension) and the fractal dimension tends to one. This is consistent with the results shown in [9] for the fractal random array.

\section{B. Further SLR Reduction: Triangular and Higher Order Generators}

The development in Section II-A was based on a uniform amplitude generator. Although this might be the optimal choice for directivity considerations, other shapes could be used to improve the SLR. Instead of convolving $M$-uniform distributions of different scales, $M$-triangular distributions could be used. Since a triangular distribution $t(z)$ can be written as the convolution of two identical uniform distributions $u(z)$, a triangular Cantor array $t c(z)$ could be generated as follows:

$$
\begin{aligned}
\operatorname{tc}(z) & =\underset{n=0}{\mathrm{X}-1} t\left(z \cdot \delta^{n}\right) \\
& =\underset{n=0}{\mathrm{X}-1}\left\{u\left(z \cdot \delta^{n}\right) * u\left(z \cdot \delta^{n}\right)\right\} .
\end{aligned}
$$

The corresponding array factor $T C(\psi)$ can be written now as

$$
\begin{aligned}
T C(\psi) & =\prod_{n=-0}^{M} T\left(\frac{\psi}{\delta^{n}}\right) \\
& =\prod_{n=-0}^{M}\left[U\left(\frac{\psi}{\delta^{n}}\right)\right]^{2} \\
& =\left[\prod_{n=-0}^{M} U\left(\frac{\psi}{\delta^{n}}\right)\right]^{2}
\end{aligned}
$$

which implies that the SLR has been doubled with respect to the uniform Cantor set $C(\psi)$. Equation (16) also implies that the triangular Cantor array is equivalent to the convolution of 


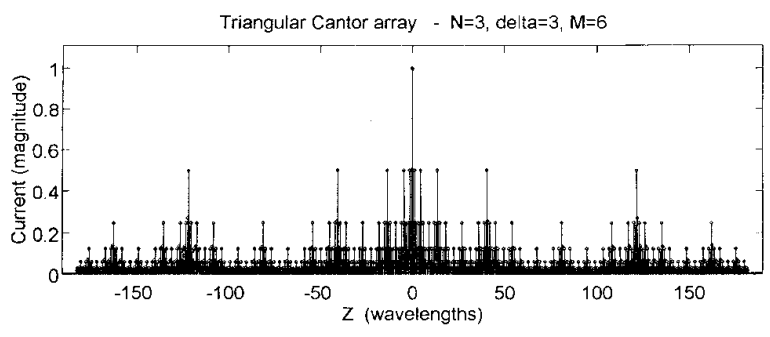

(a)

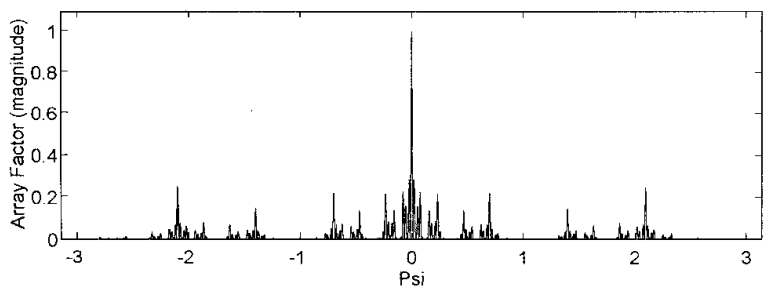

(b)

Fig. 6. Triangular Cantor array generated with six iterations: (a) the $\log$ period is $\delta=3$ and the generator is a three-element triangular array and (b) the corresponding pattern is the squared version of that in Fig. 2(a) (the SLR is doubled in a $\mathrm{dB}$ scale).

two equally-uniform Cantor arrays, an alternative procedure for its construction algorithm. The shape and the pattern of this triangular Cantor array are shown in Fig. 6.

Although this construction scheme may be useful for SLR improvement, it again introduces the inconvenience of nonuniform-amplitude distribution of the elements. Again, this problem could be solved by substituting the triangular generator by a fractal (Cantor) generator that would approach the same basic pattern. However, both solutions have a great inconvenience - the large number of elements of the array.

\section{Fractal Radiation PATtERnS}

In the previous section, a fractal analysis and design of several array factors has been developed. Fractal-element distributions have shown to be useful for designing low sidelobe array factors with a uniform-amplitude distribution of elements. On the other hand, these fractal arrays have shown some interesting similarity properties at several wavelengths, however, these similarity properties do not satisfy some of the requirements one would desire for a frequency-independent array: the directivity and the main-lobe width are not held constant at each band. In general, one would like to have an array factor which had the same shape at different scales to keep the same radiating parameters at several wavelengths. This leads to the approach presented in this section-the design of fractal array factors.

The patterns designed in this section are based on a family of self-similar curves known as Koch curves [6], [7], [15]. The pattern-construction algorithm is quite similar to that of the Koch curves, but is modified to provide a functional form. The shape and the principle of work of these kind of array factors is summarized in Fig. 7.

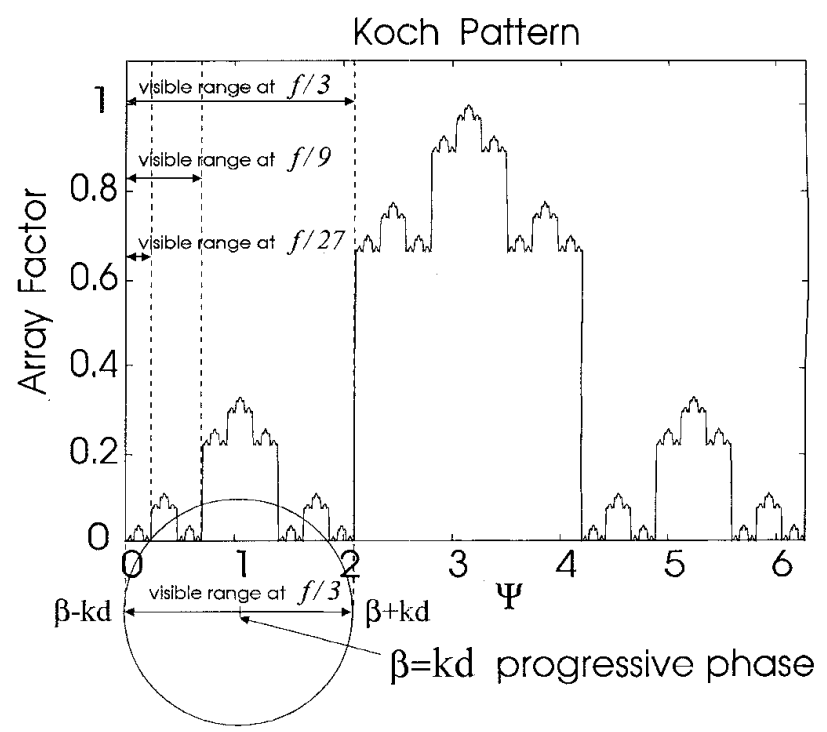

Fig. 7. The Koch-array factor. The curve keeps its similarity properties at six different scales (it has been constructed with six iterations, $M=6$ ). By adding a progressive phase $\beta=k d$, the visible range is always centered at a secondary lobe that has the same shape as the total pattern. The frequency change by a factor $\delta=1 / 3$ reduces the visible range around this similar subpattern.

The main feature of this pattern is that each lobe of the curve is equal to the whole pattern. When the array radiates at a longer wavelength, the visible range is reduced and only a fraction of the whole array factor appears in the radiation pattern. Thus, if we were able to design an array with an array factor as the one in Fig. 7, and if the visible range could be reduced around one of the secondary lobes, the resulting visible pattern would be the same as the original one. The visible range can be centered to any arbitrary point of the $\psi$ domain by adding a progressive phase $\beta(4)$ to the phase required for each element to synthesize the corresponding array factor. It can be seen that if one takes such a progressive phase to be

$$
\beta=k d
$$

the visible range will cover the interval $\{0,2 k d\}$ at any frequency. Hence, for the particular case of Fig. 7, a frequency reduction by a factor of $\left(\frac{1}{3}\right)^{n}$ would reduce the visible range around a secondary lobe which has the same shape as the whole pattern. In other words, we would have an array factor with the same radiation parameters for a set of bands spaced a factor of $\frac{1}{3}$. It is also interesting to point out that although the progressive phase in (17) is usually intended for endfire arrays, the Koch patterns are designed here to radiate in the broadside direction.

It should be noticed that, although the arrays just described would have a similar radiation pattern at several bands, the pattern magnitude is reduced when the operating wavelength is increased. That means that for the same current distribution, the electric-field intensity is reduced at lower bands or, in other words, neither the radiation resistance nor the radiation efficiency are held constant through each band. This is an 


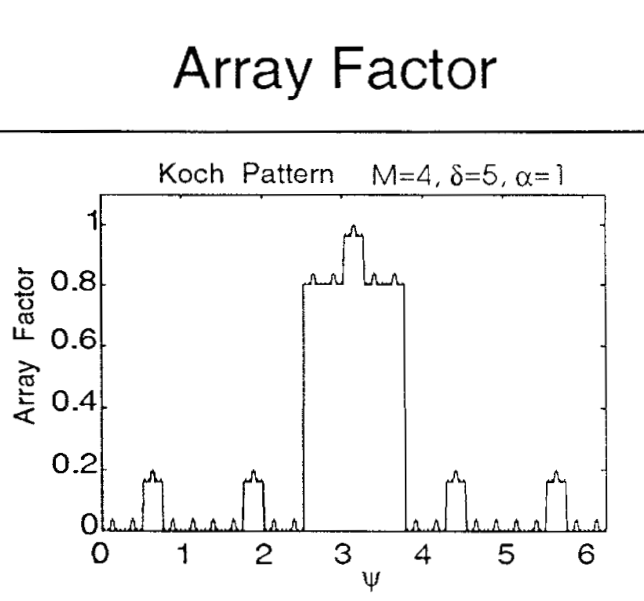

\section{Current Distribution}
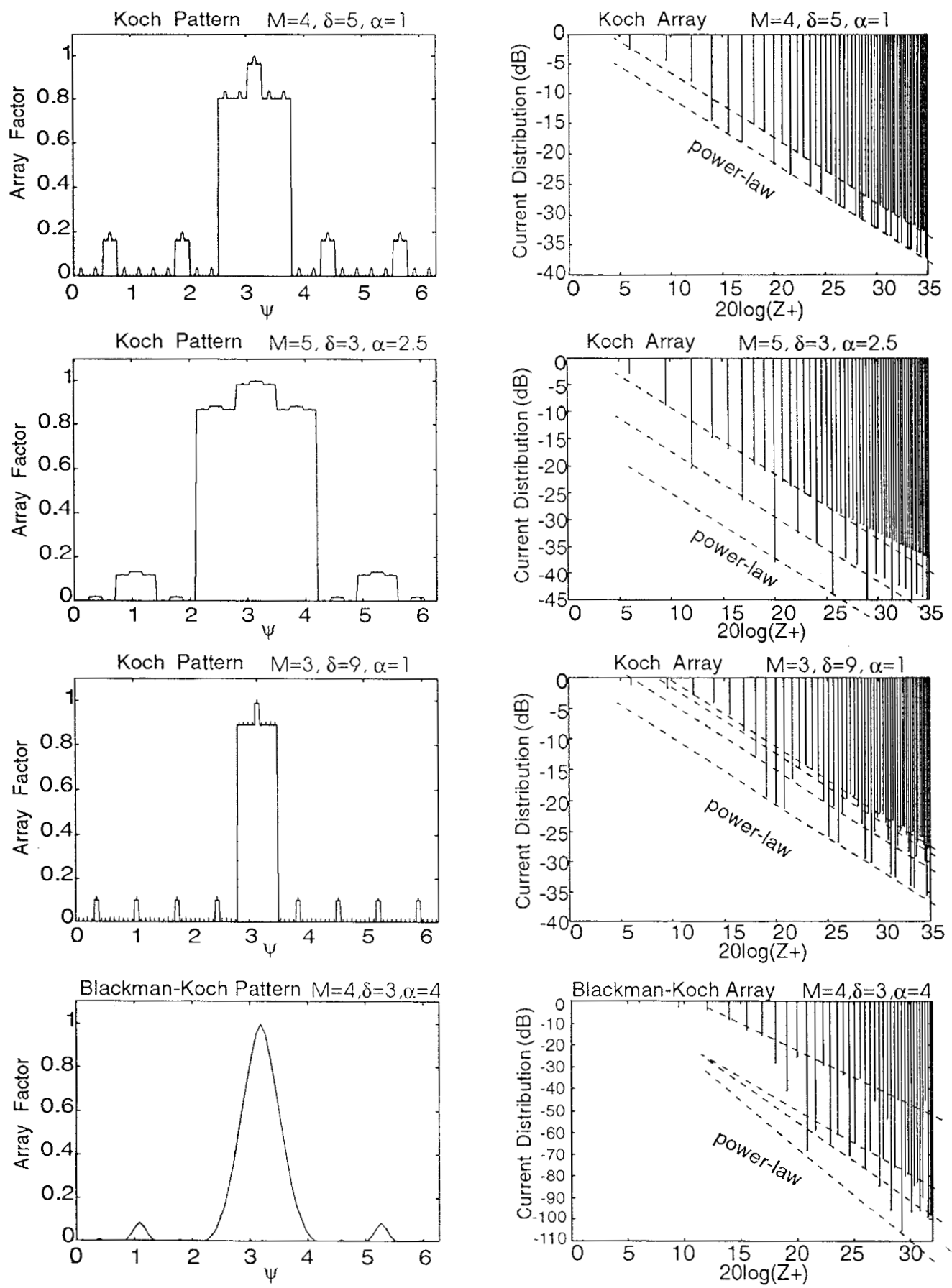

Fig. 8. Koch patterns are conformed with arrays constructed by interleaving hyperbolic distributions. The right-hand side of the array current distribution is plotted for each pattern on the left column.

intrinsic constraint of such an array design which should be faced in the physical implementation of the array.

Once the fractal-array factor has been defined to have a multiband behavior, the relative current distribution between elements that would generate such a fractal pattern has to be derived. This distribution can be numerically computed by taking the inverse Fourier transform (IFT) of the Kocharray factor. Fig. 8 shows several configurations of Koch 


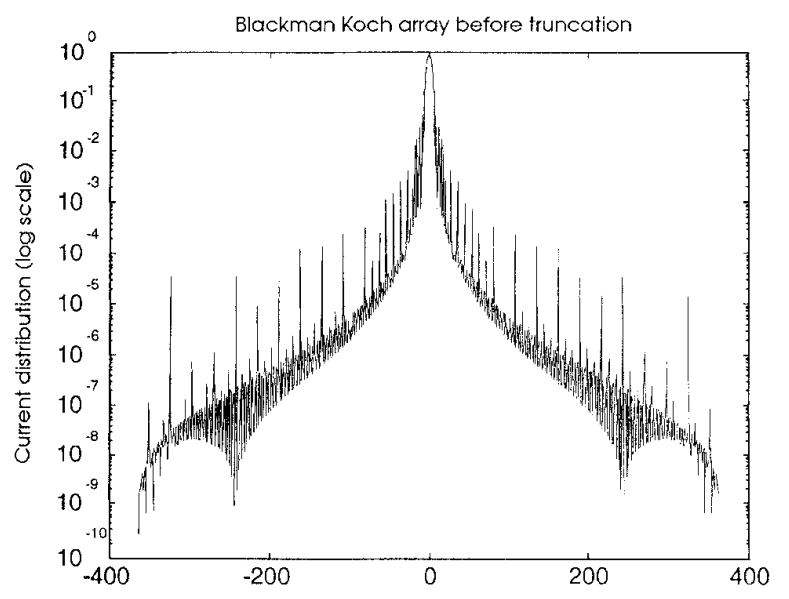

Fig. 9. Current distribution for the Blackman-Koch array logarithmic scale. The main construction parameters are $M=6, \delta=3$, and $\alpha=4$. The reduction factor $\alpha=4$ has been chosen here to improve the SLR with respect to the previous case on Fig. 7. See the corresponding pattern on the bottom case of Fig. 8 .

arrays with the corresponding current distribution. Again, $\delta$ is the log period or band ratio, $M$ is the number of iterations (and the number of bands), and $\alpha$ is an amplitude weight factor that adds an extra degree of freedom in the pattern design as it is discussed in the next subsection. Only half of the right-side of the array current distribution is displayed since it is symmetrical around its central element. The main characteristic of such current distributions is their power-lawlike shape; this feature is shared by many fractal functions, such as the bandlimited Weierstrass function [9]. The selfsimilarity properties of these array patterns are based on the scaling properties of the Fourier transform (FT) which state that if a function such as a power-law function is self-similar, so will be its counterpart in the spectral domain. In antenna theory terminology, that means that a current distribution $f(z)$ which holds the property

$$
f(z)=\delta^{\beta} \cdot f\left(\frac{z}{\delta}\right)
$$

will have a self-similar pattern $F(\psi)$, that is

$$
F(\psi)=\delta^{\beta+1} \cdot F(\delta \cdot \psi)
$$

This behavior is quite different from current frequencyindependent antennas. Such antennas are based on an active region of the antenna that changes its size with frequency. On the other hand, the fractal arrays introduced in this paper assume a current distribution that does not change with frequency but has a scale-independent shape.

Another important issue concerning the computed array structure is that it requires a large number of elements $\left(3^{6}=\right.$ 729 for the array on Fig. 7). In general, the number of elements $N$ and the number of bands $M$ in the Koch array are related as

$$
\begin{aligned}
N & =(\log -\text { period })^{\text {iterations }} \\
& \equiv \delta^{M}
\end{aligned}
$$

The number $M$ of iterations used to construct the curve determines the number of times the curve will look similar under a $\delta$ factor scaling transformation. In other words, $M$ is the number of bands or log periods in which the array will have a similar pattern. Hence, there is a trade-off between the size of the array and the number of operating bands. Of course, here arises what can be an intrinsic limitation of these arrays: the number of elements grows exponentially with the number of log-periodic bands. Since all different Koch patterns that have been analyzed [14] have the common characteristic of concentrating the most important current contribution around the central element, one could think that the number of elements could be reduced by merely truncating the array at its tips. However, it can be readily seen that this procedure would limit the multiband behavior of the array. An array truncation is equivalent to a spatial windowing of the structure, which is equivalent to low-pass filtering the pattern in the $\psi$ domain. Therefore, the array factor is smoothed and the pattern loses its characteristic lobe structure which is the base of its multiband behavior [14]. A deeper analysis of the Koch array structure will help in both understanding its behavior and reducing the number of elements.

Analysis of the Array Element Distribution for Koch-Pattern Conformation: A key point for understanding the array current distribution derived from the fractal patterns is the Kochpattern construction algorithm itself. Let us take a periodic pulse train in the spatial-frequency domain $\psi$ and scale its width by a factor $\delta$ and its amplitude by a factor of $\alpha \delta$. After iterating this scheme $M$ times, the $M$-resulting patterns are added, obtaining a Koch pattern such as the one in Fig. 7. In particular, for the pattern on Fig. 7, a rectangular pulse and a $\log$-period $\delta=3$, and an amplitude factor $\alpha=1$ was chosen for generating the pattern with $M=6$ iterations.

The analytical expression for each generating pulse train can be written as

$$
F(\psi) * \sum_{n=-\infty}^{\infty} \delta\left(\psi-n \psi_{T}\right)
$$

where $F(\psi)$ is the single pulse function, which, in general could be taken to have any arbitrary shape such as a rectangular window or a Blackman window, and $\psi_{T}$ is the period of the pulse train. From (21), the analytical expression for the Koch pattern $K(\psi)$ after adding the $M$-scaled pulse trains is

$$
\begin{aligned}
K(\psi)= & \sum_{p=0}^{M-1} F\left(\delta^{p} \psi\right) \\
& *\left\{\frac{1}{\alpha^{p} \delta^{p}} \sum_{n=-\infty}^{\infty} \delta\left(\psi-n \frac{\psi_{T}}{\delta^{p}}\right)\right\} .
\end{aligned}
$$

Several combinations of $\alpha, \delta$, and $M$ are essayed in the arrays of Fig. 8. A rectangular generating pulse is chosen on the first three examples, and a Blackman window on the last one. Once an expression for the Koch pattern has been derived, an expression for the Koch-array element distribution $k(z)$ can 
be easily found by taking the IFT of (22)

$$
\begin{aligned}
k(z)= & \frac{1}{\psi_{T}} \sum_{p=0}^{M-1} \frac{1}{\delta^{p}} f\left(\frac{z}{\delta^{p}}\right) \\
& \quad\left\{\frac{1}{\alpha^{p}} \sum_{n=-\infty}^{\infty} \delta\left(z-n \cdot d \cdot \delta^{p}\right)\right\} .
\end{aligned}
$$

The train of delta functions in (23) samples the current distribution at the discrete set of points $z=n \cdot d \cdot \delta^{p}$ where the array elements are located. Hence, taking into account that

$$
\begin{aligned}
\psi_{T} & =k d \\
& =\frac{2 \pi d}{\lambda}
\end{aligned}
$$

one can write

$$
\begin{aligned}
k(z)= & \frac{\lambda}{2 \pi d} \sum_{p=0}^{M-1} \\
& \cdot\left\{\left(\frac{1}{\alpha \delta}\right)^{p} \cdot \sum_{n=-\infty}^{\infty} f(n d) \cdot \delta\left(z-n \cdot d \cdot \delta^{p}\right)\right\}
\end{aligned}
$$

which gives an insight into the shape of the resulting array; the Koch array is a superposition of $M$ arrays that have the same element distribution but a wider spacing between elements, depending on the iteration stage to which they belong. That is, the elements are uniformly spaced within the same array, but the spacing changes at each subarray by a factor $\delta^{p}$. When the arrays are added, some elements might fall at the same position as other elements from the other arrays; in such a case, the result is a single element whose weight is the sum of the weights of all the elements that would fall at that point. In particular, it can be seen that all the arrays have a common element at

$$
z=n \cdot d \cdot \delta^{M-1}
$$

Equation (25) can give an insight on the power-law shape of the current distribution that generates the Koch pattern in Fig. 7. For this particular case, the squared pulse generator has an inverse transform

$$
f(z)=\frac{\sin \left(\frac{\pi}{d \cdot \delta} z\right)}{\pi z}
$$

which gives the shape of all the $M$ subarrays that conform the Koch array. The weight of each element can be easily found in our sampling (27) at $z=m \cdot d$. It can be seen that for the case we have been studying $(\delta=3$ )

$$
\begin{aligned}
|f(m d)| & =\left|\frac{\sin \left(\frac{\pi}{3} m\right)}{\pi m d}\right| \\
& = \begin{cases}\left|\frac{\sqrt{3}}{2 \pi d}\right| \cdot \frac{1}{|m|} & m \neq 3^{w} \\
0 & m=3^{w}\end{cases}
\end{aligned}
$$

which is, in absolute value, a power-law (hyperbolic) function of the index element $m$. Another important property can be seen if we realize that $(28)$ is null for those $m$ such that

$$
m=\delta^{W}
$$

with $W$ being an integer. From this property, one concludes that all the subarrays contribute to the weight of the central element, but they do not overlap at any other point since the nulls of each array are filled by an element of an array corresponding to the next iteration stages. Therefore, the global array obtained after $M$ iterations can be seen as an array composed by interleaving the elements of $M$-equal arrays at $M$-different scales. The result for the particular case $\delta=3$ and a square pulse generator, is an equally-spaced array with a hyperbolic distribution of the element-current magnitudes.

Two important conclusions can be derived from the analysis of the generalized Koch-array $k(z)$ and the particular case we have just studied. First, the shape of the $M$-superimposed subarrays depends on the shape of the pulse generator. Second, the superposition of the subarrays might result in the confluence of many elements in a single location or might result in an interleaving of the elements. As will be shown in the following subsection, both conclusions will help in the reduction of the number of elements of the Koch array.

The Blackman-Koch Array: A Further Simplification of the Fractal-Pattern Array: Since the array current distribution is basically a superposition of the inverse transforms of the pulse generator, it should be chosen a pulse generator with a low side-lobe level transform to allow a better truncation of the Koch arrays just shown. The Blackman window is characterized for having low side-lobes in the transformed domain. Therefore, one could chose a train of Blackman pulses to generate the Koch patterns instead of the rectangular ones. The results of applying such a technique are shown in the bottom case in Fig. 8 .

It can be noticed that the pattern results in a smoother shape that keeps the same similar properties of the Koch-array factor of Fig. 7. The main advantage of this pattern is that the array-relative current distribution has lower side-lobes and a better confinement around the central elements (Fig. 9). Also, the logarithmic plot of the current distribution reveals some important isolated current peaks well beyond the center of the array. One should expect a significant contribution of these isolated elements to the global-pattern conformation. Thus, instead of just truncating the tips of the array, a threshold level can be set to discern which elements are important in the pattern synthesis and which are not. The result is that the array structure can be reduced to only the 75 elements (as opposed to 729) with a higher current contribution and still keep its self-similar behavior at five bands through a whole $81: 1$ frequency range. The resulting array is no longer a uniformly-spaced array since the main 75 current elements are not placed together near the midpoint of the array. Thus, some elements are placed further from the origin than in the truncation scheme which means that faster variations will appear in the dual domain (the pattern domain). This explains why this scheme can better keep the multiband behavior in a larger number of bands than the truncation scheme: the further 
the elements are placed from the origin, the finer will be the resulting lobe structure which will allow the pattern to keep the same shape for a further reduction of the visible range.

\section{CONCLUSION}

A novel approach to the design of frequency-independent radiating systems has been presented in this paper. Fractal structures are used in the design because of their self-similarity properties. The effort has been focused in describing a technique to design low side-lobe and multiband arrays, which has always been difficult due to the sensitivity of most current design techniques to variations on the operating wavelength.

Two main approaches have been followed in Sections II and III, respectively: the placement of the array elements on a fractal set of points (the Cantor set) and the design of array factors with a fractal (Koch) shape. Although the Cantor arrays have been shown to have similar patterns at several bands, some important properties such as mainlobe width and directivity are not held constant through the bands. On the other hand, such structures have shown to be useful to synthesize low side-lobe patterns with uniform amplitude current distribution arrays. The Koch-array factors (designed in Section III) do keep the same directivity, lobe structure, and SLR at each operating band. A Koch pattern designed by using a Blackman window generator can be conformed with 75 elements, resulting in an array factor that would operate at five bands, covering a total $81: 1$ frequency range. Such an array would present a multiband behavior rather than a frequency-independent behavior and its radiation resistance is not held constant through the bands. The current distribution that would generate such a pattern can be seen as an interleaving of power-law arrays, which present some interesting self-similarity properties as well.

\section{ACKNOWLEDGMENT}

The authors would like to thank Dr. P. E. Mayes from the Electromagnetics Laboratory, University of Illinois, UrbanaChampaign, and Dr. A. Cardama, Dr. J. M. Rius, and the entire Antenna, Microwave, and Radar Group of the Polytechnic University of Catalonia, Barcelona, Spain, for their encouragement and helpful suggestions to this work.

\section{REFERENCES}

[1] P. E. Mayes, "Frequency-independent antennas and broad-band derivatives thereof," Proc. IEEE, vol. 80, no. 1, Jan. 1992.

[2] P. E. Mayes, G. A. Deschamps, and W. T. Patton, "Backward-wave radiation from periodic structures and application to the design of frequency-independent antennas," Proc. IRE, vol. 49, pp. 962-963, May 1961.

[3] G. A. Deschamps and J. D. Dyson, "The logarithmic spiral in a singleaperture multimode antenna system," IEEE Trans. Antennas Propagat., vol. AP-19, pp. 90-96, Jan. 1971.

[4] V. H. Rumsey, Frequency Independent Antennas. New York: Academic, 1966.
[5] R. L. Carrel, "Analysis and Design of the log-periodic dipole antenna," Doctoral thesis, Dept. Elec. Eng., Univ. Illinois, Urbana-Champaign, 1961.

[6] B. B. Mandelbrot, The Fractal Geometry of Nature. San Francisco, CA: W. H. Freeman, 1983.

[7] M. F. Barnsley, R. L. Devaney, B. B. Mandelbrot, H. O. Peitgen, D. Saupe, R. F. Voss, Y. Fisher, and M. McGuire, The Science of Fractal Images. New York: Springer-Verlag, 1988.

[8] D. L. Jaggard, "Prolog to special section on fractals in electrical engineering," Proc. IEEE, vol. 81, no. 10, pp. 1423-1427, Oct. 1993.

[9] D. L. Jaggard, "On fractal electrodynamics," in Recent Advances in Electromagnetic Theory, D. L. Jaggard and H. N. Kritikos, Eds. New York: Springer-Verlag, 1990, pp. 183-224.

[10] Y. Kim and D. L. Jaggard, "The fractal random array," Proc. IEEE, vol. 74, no. 9, pp. 1278-1280, Sept. 1986.

[11] D. L. Jaggard and T. Spielman, "Triadic cantor target diffraction," Microwave Opt. Technol. Lett., vol. 5, no. 9, pp. 460 466, Aug. 1992.

[12] C. Goutelard, "Fractal theory of large arrays of lacunar antennas," in Electromagn. Wave Propagat. Panel Symp. (AGARD.CP-528), June 1992, pp. 35/1-15.

[13] H. Jasik, Antenna Engineering Handbook. New York: McGraw-Hill, 1961 , pp. $2.10-2.13$.

[14] C. Puente, "Fractal design of multiband antenna arrays," Elec. Eng. Dept., Univ. Hllinois, Urbana-Champaign, ECE 477 term project, Dec. 1993.

[15] H. Jones, D. E. Reeve, and D. Saupe, Fractals and Chaos, A. J. Crilly, R. A. Earnshaw, and H. Jones, Eds. New York: Springer-Verlag, 1990.

[16] X. Sun and D. L. Jaggard, "Wave interactions with generalized Cantor bar fractal multilayers," J. Appl. Phys., vol. 70, no. 5, pp. 2500-2507, Sept. 1991.

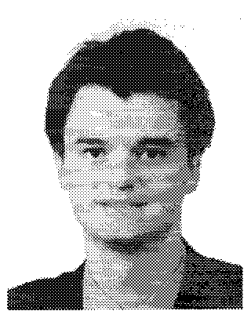

Carles Puente-Baliarda (S'91-M'93) was born in Badalona, Spain, in 1968. He received the Ingeniero degree in telecommunications engineering from the Polytechnic University of Catalonia, Barcelona, Spain, in 1992, and the M.S. degree from the University of Illinois, Urbana-Champaign, in 1994.

In 1992, he got a grant from the CIRIT (Interdepartamental Commission for Research and Technological Innovation) of the Catalonian government and joined the Electro-Optic Systems Laboratory (EOSL) at the University of Illinois, Urbana-Champaign. He worked there in laser velocimetry and lidar techniques until 1994. Since 1994, he has been Assistant Professor at the Department of Signal Theory and Communications (TSC) of the Polytechnic University of Catalonia, while working toward the Ph.D. degree. His current research interests are fractal antennas, lidar, laser systems, and optical communications.

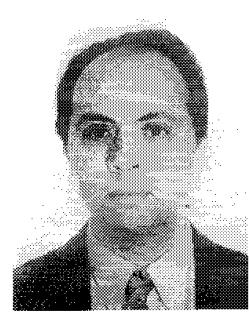

Rafael Pous (''89-M'93) was born in Barcelona, Spain, in 1964. He received the Ingeniero degree in telecommunications engineering and the Licenciado degree in computer science from the Polytechnic University of Catalonia, Barcelona, Spain, in 1988. He received the M.S. degree in electrical engineering from the University of Massachusetts, Amherst, in 1989, and the Ph.D. degree in electrical engineering from the University of California, Berkeley, in 1992. He was awarded the Fulbright Scholarship and the Schlumberger Fellowship during his studies. In 1993, he joined the Telecommunications Engineering faculty of the Polytechnic University of Catalonia, Barcelona, Spain, where he is currently employed. His research interests are in the areas of computational electromagnetics and applied superconductivity. 